\title{
Traffic Sign Shape Detection and Classification based on the Segment Surface Occupancy Analysis and Correlation Comparisons
}

\author{
Tomislav KESER, Ivan DEJANOVIĆ
}

\begin{abstract}
This article addresses the issue of traffic sign recognition. It contributes to a growing body of research done by the automotive industry due to a necessity for ensuring better safety on the roads. This paper presents a novel method for traffic signs recognition. The implementation of the whole process of traffic sign recognition has a step-wise nature but the novelty is introduced into the traffic sign shape detection stage. The method is based on a new approach for traffic sign shape recognition based on the image content occupancy analysis. Further, the traffic sign content classification is based on a simplistic relational correlation analysis. The tests were performed on image data comprising various roads and lighting conditions. The test includes different sizes of templates used in the correlation comparison method. The results are presented in a manner of successfulness of the correct recognition.
\end{abstract}

Keywords: correlation comparison; image analysis; occupancy analysis; traffic sign detection; traffic sign recognition

\section{INTRODUCTION}

The automotive industry has constantly been making tremendous efforts in conducting research and projects related to the development of assisted driving technologies. Some of these technologies niches focus on the problem of automatic recognition of traffic signs [15]. Despite the fact that the most advanced vehicles are equipped with these systems, a man still plays a major role to ensure the safety in the traffic environment as described in [6, 7]. One of the ultimate goals addresses the issue of developing an autonomous or automated vehicle that will manage driving without the necessity for a human action. Such vehicles need to be equipped with sensors used for "sensing" the environment and driving within predefined paths. Sensor systems that can be used for "sensing" the environment are radars, light radar (lidars), GPS, computer vision and advanced control systems. Such systems interpret sensory information and identify appropriate navigational paths as well as obstacles and appropriate traffic signs. The ability to update maps based on sensory input allows a vehicle to monitor its position even when conditions are challenging or when the environment is not drawn on maps (like traffic signs). Currently, these vehicles exist as demonstration systems and prototypes.

In the traffic sign recognition research field, numerous methods and approaches are used. Segmentation by colour is often used to detect areas where traffic signs appear like RGB [3, 4, 8, 15] or HSV/HSI colour model $[5,11,12]$. Other methods that track corners and edges were used in the shape analysis [9]. Further, recognitions can be achieved with neural networks [11, 14] or template matching [4, 15]. Some features, such as Histogram of Oriented Gradients (HOG) and features with linear Support vector machine (SVM) classifier are successfully used for a fast computation in traffic sign recognition process [3, 12]. Also, the Kalman filter can be used for predictions of traffic sign candidates in video frames, so they can be successfully tracked in real-time situations [6].

In this paper, we present a method based on the segment surface occupancy analysis. That method is novel, to the authors' knowledge, and there are no similar approaches or applications of it. Our goal is to elaborate on the method and validate its applicability.

This paper is organised in four main sections. The first section (Section 2) gives an overview of the method used in this paper. Also, an algorithm flow of the programme and its implementation is presented. In Section 3, sign detection and localisation followed by shape and content recognition are described. The experimental results are presented in Section 4. Finally, the conclusions and suggestions for further research are given in Section 5.

\section{TRAFFIC SIGN CLASSIFICATION AND RECOGNITION}

Solution starter is based on the detection and recognition of red and blue traffic signs. Widely known and frequently used approaches are colour segmentation, object separation and a grey scale analysis [8,9]. Groups (classes) of traffic signs that belong to the area of the analysis are explicit order (mandatory) signs, information signs and warning signs. In Croatia, these classes of signs constitute the majority of traffic signs and are necessary in traffic systems in terms of the traffic system safety [10]. In addition, these signs are characterised by the colour and shape which are important for signs classification. Blue signs have a square, rectangular and circular shape and red signs have the shape of a triangle, octagon and circle.

Therefore, traffic signs can be described by three properties:

1) colour (red, blue, etc.);

2) shape (triangle, circle, square, rectangle and octagon);

3) the inner content of the sign which consists of symbols and text.

Therefore, in order to properly detect and recognise a traffic sign, it is necessary to carry out an analysis for all three properties of the sign.

\subsection{Method Structure and Algorithm Flow}

An algorithm analysis flow and structure of this method is presented in Fig. 1. This flowchart shows important steps and their logical flow that defines the operational unity of the whole process. The flowchart 
(Fig. 1) for the algorithm of traffic signs recognition consists of three parts:

1) detection - where the colour detection of traffic sign candidates takes place;

2) classification - where the shape selection occurs.

3) recognition - where the template matching occurs.

Numerous authors use a basic algorithm form which consists of detection, classification and recognition phases [2-6, 13]. For example, in [12], the algorithm logical flow is similar to our method flow. It differs in the concept of the recognition stage which is actually one part of the classification phase. Even if phases are named similarly or equally, they use different methods, as in this particular example, even if both methods use the colour segmentation approach as a detection method. We use different colour models like RGB and HSV. The same is applied in other stages and methods.

In a detection phase, firstly, RGB images are filtered with a median filter on each chrome channel followed by a colour segmentation phase. This phase focuses on the segmentation of red and blue coloured objects of the analysed image. The result of the segmentation is a binary image of red and blue coloured and emphasised signs. With further binary image processing, characteristics important for road signs are extracted. These characteristics are the colours of the sign (for example, red in the case of a triangle or circle) and the inner content of the sign which is mostly filled with symbols or text.

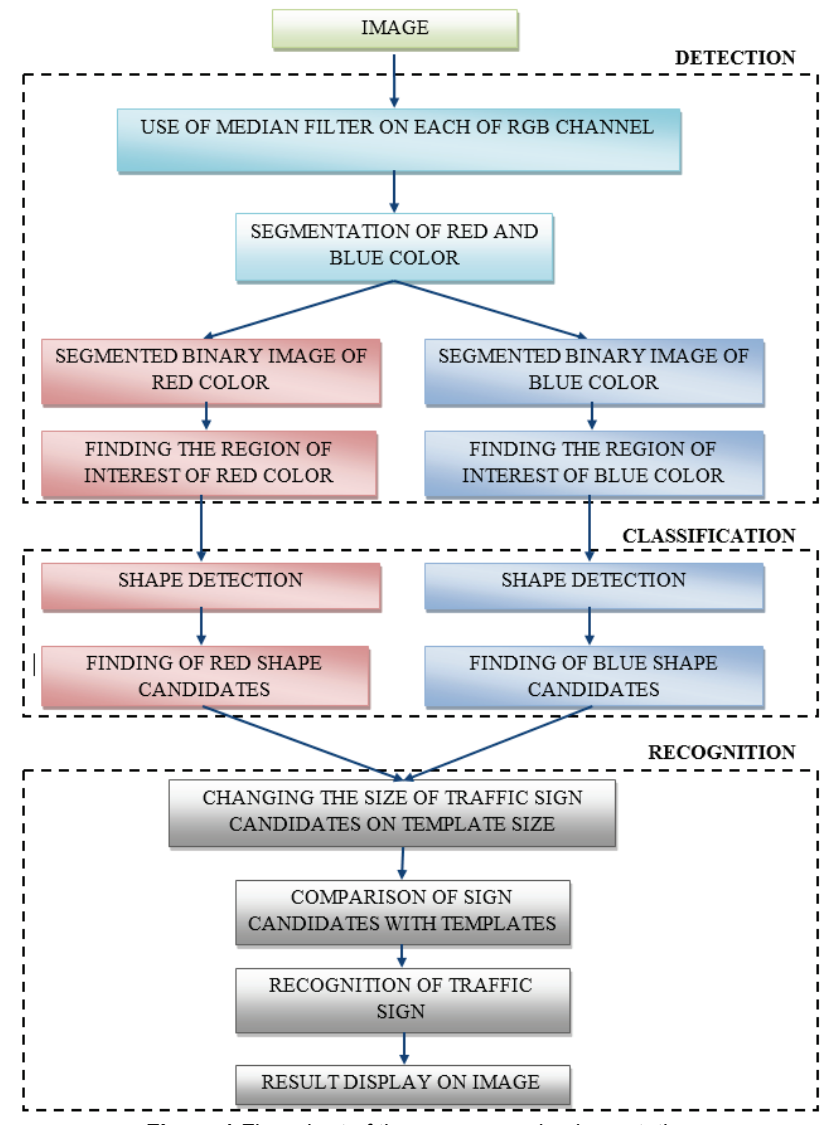

Figure 1 Flow chart of the programme implementation

A classification phase consists of filtering and sorting objects that had met requirements laid down by detection according to the "colour" feature. On the other hand, traffic signs are characterised by certain geometrical properties. So, filtering is done by using the width and height of a rectangular frame that is placed over the sign. The height and width are approximately of the same length when they are viewed at angle $0^{\circ}$, i.e. when the traffic sign is looked at directly. In this way, candidates not meeting the requirements of width and height of a rectangular frame placed over a potential traffic sign are rejected. Looking at a traffic sign at some angles gives different ratio values of width and height. Classification of objects or shape detection takes place according to the ratio of the sign area (pixels of a binary object) and the area of the corresponding rectangular frame. Hence, objects are classified in the form of a triangle, circle, square and octagon.

Candidates who passed the sign checks on colours and shapes features are used at the recognition stage. Then, these candidates are trimmed down to a size given by sing templates. In this way, the candidate of a traffic sign is prepared for the comparison with the templates. The results corresponding to a certain level of similarity are accepted, while other results are rejected. Once the individual candidate of a traffic sign is recognised, a rectangular frame is drawn on the image with the corresponding correlation coefficient denoted.

\section{SHAPE DETECTION AND CLASSIFICATION}

Implementation of the traffic sign recognition algorithm is carried out in the MATLAB programming environment by using image processing functions (Image Processing Toolbox) and custom made methods. At the moment, the authors are focusing to implement the method, prove its working concept and justify its applicability. Neither a performance analysis and any kind of algorithm optimisations nor the comparisons with other goal-similar methods are done. As previously stated, the presented method is divided into three functional parts (steps) which are elaborated on in the following section.

\subsection{Image Segmentation - Sign Detection and Localization}

The first step in the detection phase is filtering an image using a median filter. By reducing the image noise, this step improves the image prior to the segmentation phase. The median filter is a nonlinear filter whose response is based on the order of pixels contained in the image area covered by the filter. It replaces the pixel value of the centre of the mask with filter value neighbourhood pixels determined by the size ranking.

The median filter allows noise reduction with less blur than the linear smoothing filter of a similar size. It is particularly effective in reducing impulse noise such as "salt and pepper". The noise appears as black pixels in a white environment of pixels and vice versa.

The filtering results are obtained by taking into account the pixels covered by the mask of the filter whose values are ranked in size from the smallest to the largest. The value of the pixel located in the middle of this sequence is taken and placed in the central pixel neighbourhoods of the image covered by the filter mask. Filtration by the median filter included in this analysis is done by using kernel $3 \times 3$ pixels in size and takes place separately on each channel of the RGB image. 
The RGB system consists of three channels, namely red, green and blue. The combination of their values forms a colour. For the analysis of the programme work, the main focus is on red and blue colours. Red and blue colours are not built from solely pure red or blue. They contain other components of the RGB colour system. For example, red colour contains red, blue and green components. The condition is that the red colour component is predominant by its value as opposed to other components in the RGB image.

Traffic sign detection is performed in the RGB colour space by building a binary image where " 1 " represents the pixels belonging to the candidate traffic sign, binarisation respectively. The segmentation takes place on red and blue by the threshold method as follows:

\section{Binary image of red colour $=$ \\ Red channel $>$ treshold \\ $\left\{\right.$ Green channel $<k_{G} *$ Red channel, \\ Blue channel $<k_{B} *$ Red channel}

\section{Binary image of blue colour $=$}

Blue channel $>$ treshold

$\left\{\right.$ Red channel $<k_{R} *$ Blue channel,

Green channel $<k_{G} *$ Blue channel

Where the "threshold" is fixed, the number can range from 0 to 255 values for 8-bit data. In the analysis, it is set to value 40 , so that darker values of red and blue colour can be read and the " $k$ " value is in the range from " 0 " to "1". It corresponds to what proportion must be of channels RGB colour system in the main channel, i.e. how much percentage of secondary channels is in the formation of colour as opposed to the main channel. In this way, it is ensured that the main channel is dominant in the formation of a specific colour.

One example of the colour segmentation is shown in Fig. 2.

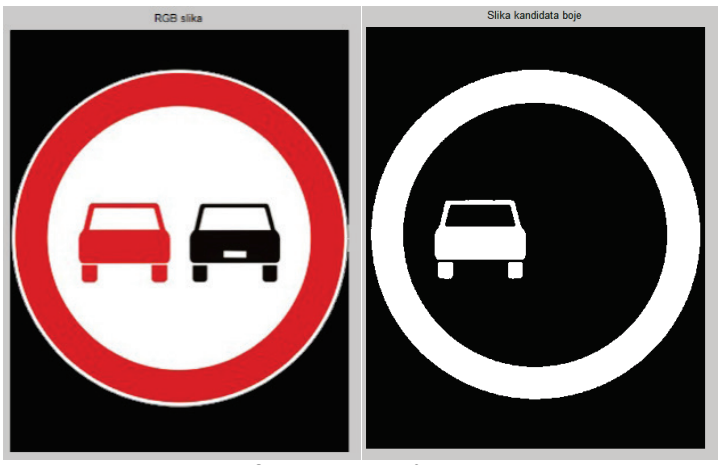

Figure 2 Segmentation of the red colour

The next step is to use morphological close operations. It is a process of dilation followed by erosion with the same structural element. With dilatation of the binary image obtained by the expansion of binary image pixels, we achieve the growth of objects. Spreading a pixel of the binary image allows the pixels which are close enough and are pixels of the traffic sign to become pixels of the same object. Erosion returns the size of the object at about the same size prior to dilation. This procedure attempts to correct imperfections of threshold methods due to different variations of lighting and various obstacles that hinder the visibility of the sign by connecting punctuated pixels of the traffic sign.

The next procedure is deleting small objects from the binary image. The process of segmentation results with binary image objects of particular colours. Objects are connected pixel values, in our case "1". In order to be able to analyse individual objects, but also to be a potential candidate for the traffic sign, it must have the necessary size of the connected pixels.

After deleting small objects, the following is the procedure for filling the interior of binary objects. These are morphological operations which are necessary for the calculation of geometrical characteristics of the object (Fig. 3, left image).

To make MATLAB "know" to distinguish objects, they must be labelled. Labelling is done on each connected pixel adjacent to each other. Labelled objects are different shades in the view which proves that they are different objects (Fig. 3, right image).
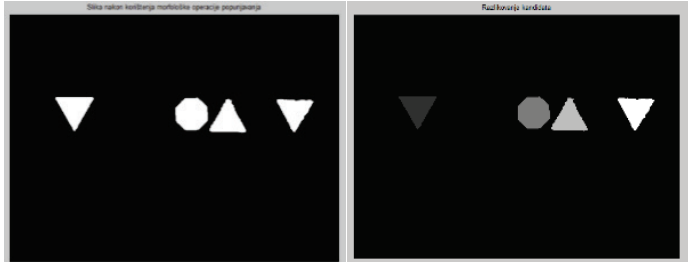

Figure 3 Binary images after filling interior and labelling objects

Note that the objects are prepared for the implementation of geometric analysis and shape detection. The parameters that need to be taken into consideration for the analysis are the bounding box (rectangular frame) parameters and the object area parameter.

The bounding box is a simple parameter for crude description of the object size. It is defined as a rectangle that is large enough to contain all the pixels of the object. It also gives a rough description of the object shape. The bounding box is a feature of object-oriented pixels processing such as the extraction of object pixels for further purposes.

The area parameter is another parameter form, i.e. another way of describing the binary objects extracted from the image data. The area in a binary image is the sum of pixels assigned to the surface, i.e. object. This means that the area of the object can be calculated simply by counting the pixels within the object.

The starting point of the image is the upper left corner which is at coordinates $(0,0)$. The first variable is $x$ coordinate which moves from left to right, and the second is y coordinate which moves from up to down. The knowledge of this principle is the starting point needed for creating the bounding box. The bounding box consists of four variables. The first two are the coordinates of the pixel $(x, y)$ located in the upper left corner of the frame. The other two variables are the width and height of the frame. The bounding box is placed over an object, so that it can make the calculation and extract four variables of the box for each object.

After getting parameters of the bounding box and calculating the objects, we crop the RGB image by the obtained coordinates. With this procedure, we get regions 
of interest of the RGB image, image segment for further analysis respectively, for example, the region of interest of red or blue colour. These regions are potential candidates for traffic signs.

The next step is creating a mask of regions of interest by cropping objects of the binary image and switching it to the grayscale form. In that form, we can implement multiplication with each channel of the appropriate cropped RGB image. This process creates the mask of the RGB image (Fig. 4, left image).

Cropped objects of the RGB image may contain areas that are not signs, therefore, this procedure needs to be carried out and set them as background, i.e. "0".

Further processing is performed in order to extract symbols within the inner content of the traffic sign. We again use the threshold method within the obtained RGB image mask in order to obtain a binary image of the region of interest. Morphological operations of filling the interior are used upon obtaining the binary image.

To obtain the inner area of the traffic sign (Fig. 4, right image), we perform the subtraction operation of the binary image and its version with the filled interior. The resulting image after subtracting has the black value as "-1" and the white value as "0". This is corrected by adding " 1 " to each pixel value. In this way, we ensure that the black is " 0 " and the white is " 1 ".

Further step is making complement of that binary image (Fig. 5, left image). The resulting image has the value of " 1 " for the inner content of the traffic sign and the pixel values outside the inner content of the sign are " 0 ". This form is used to get the binary mask and conduct the search of internal traffic sign symbols. In order to do this, the morphological operations of filling the interior are done. Their purpose is to make 8-bit binary masks that will once again carry out multiplication with the previously obtained cropped RGB image.

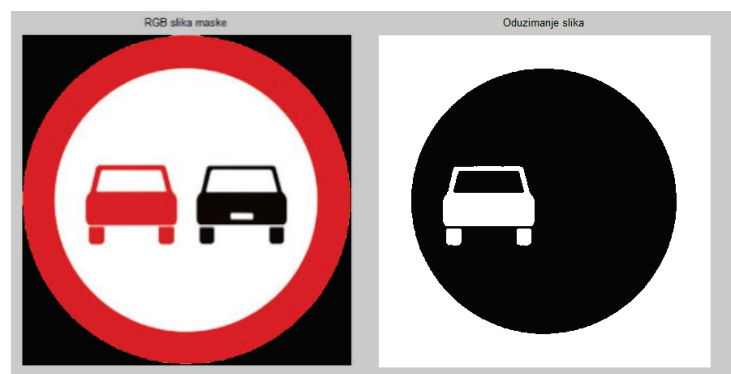

Figure 4 Mask of the RGB image and binary image obtained by subtraction of binary images

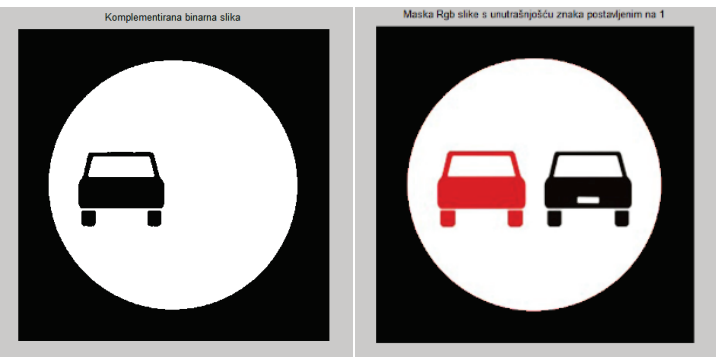

Figure 5 Binary image complement and the mask of RGB image with the inner content

The image that is obtained by this method contains only the elements (placed in the interior of the sign) of the
RGB image, i.e. all the others are set to "0" as shown on the right image in Fig. 5.

The threshold method by red, blue and black colour (Fig. 6) is employed. Black is a constituent colour for the majority of symbols within the traffic signs and its search is necessary.

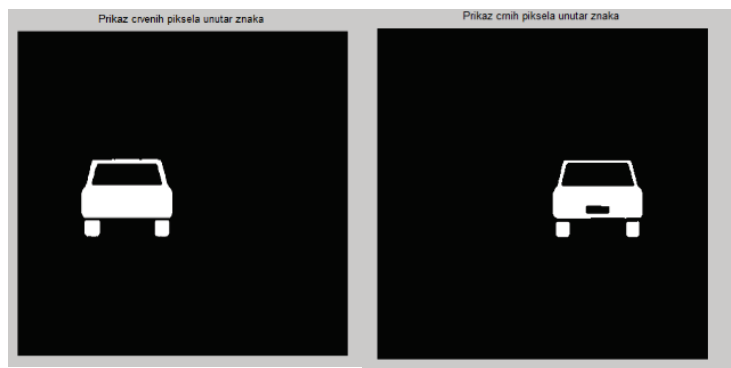

Figure 6 Binary images of the inner content

Once a binary image is obtained by the threshold of red, blue and black colours, we use the morphological operations of filling the interior of the symbols. The operation is done to fill the interior of the object symbols for more accurate processing, but also due to "holes" within the object symbols resulting from the imperfections of the method threshold (Fig. 7).

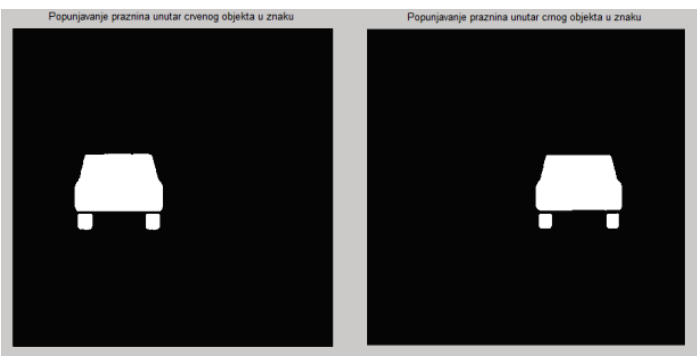

Figure 7 Filled binary images of the inner content

Having received a more compact objects form within the sign, it is necessary to use a morphological closing operation. This operation connects tiny segments within a sign if they are close enough. This will result in a form that is much easier to analyse (Fig. 8).

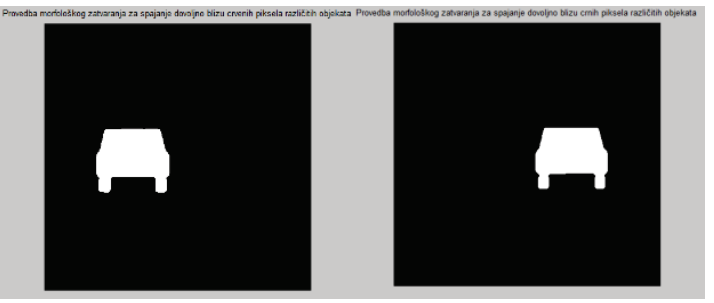

Figure 8 Binary images of the inner content after the closing operation

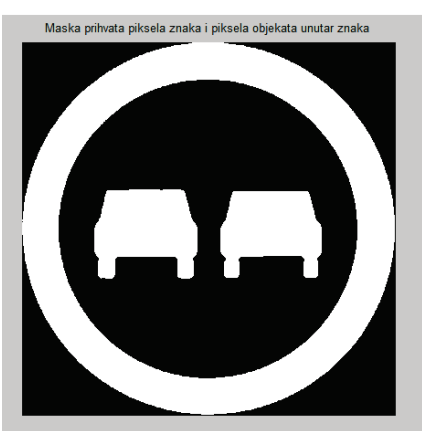

Figure 9 Final binary image 
The latter procedure is used by creating masks of " 0 " that accept the values. Then, the values of the internal elements, along with the elements of the outer circle of the sign, are added within the acceptance mask to form a total binary image of the road sign suitable for the analysis (Fig. 9).

\subsection{Shape Recognition and Classification}

The shape selection of sign candidates occurs in the classification stage. The criterion for the selection of segmented traffic signs is adopted according to the size and proportion of the object. This method is called a segment occupancy density analysis.

Two criteria are taken into account. The first criterion is the aspect ratio of the bounding box (analysis segment), which is placed on the binary image objects. The second criterion is the ratio of the sign area and the bounding box area, occupancy density, respectively.

Their values in the analysis are obtained experimentally by measuring different forms of traffic signs under different viewing angles. As a reference angle, $0^{\circ}$ is used, i.e. an angle that looks straight onto the surface of the traffic sign. The bounding box is an essential part of the analysis of traffic signs because it is used for the selection of geometric shapes. Every traffic sign has geometric properties that distinguish it from other signs.

The first part of the classification takes place in rejection of objects that do not belong to the predetermined range of the aspect ratio of the bounding box. This is obtained in a way that the frame is set to the object and performs the calculation of the width and height ratio of the frame. The range is usually between 0,9 and 1,1 for all traffic signs except the rectangle that is approximately at the value of 0,66 at reference angle $0^{\circ}$.

The second part of the shape parameter is obtained in a similar manner as the first parameter. The bounding box is placed on the object. The ratio of the object area (the number of pixels within the object) and the area of the bounding box (the product of the width and height of the frame), the surface occupancy respectively is calculated. Their ratios and occupancies are typical for each traffic sign; they are different and distinctive. Only in the case of a circle and octagon, we find the similarity of values. Therefore, in the analysis of the traffic sign, they are placed in the same group of signs entitled "circle". The visual display of the traffic signs with their assigned bounding boxes is shown in Fig. 10.

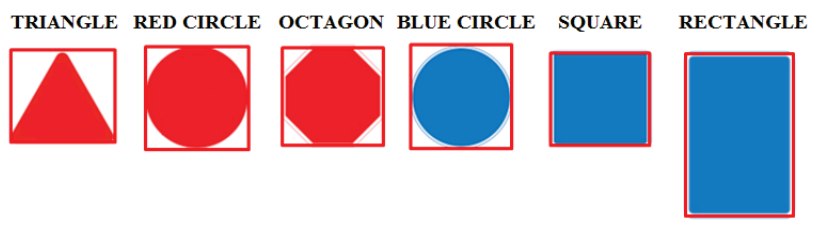

Figure 10 Traffic signs with the associated bounding boxes

One example of obtaining the ratio of the object area and the surface of the bounding box, occupancy, is shown in the following calculation for the parameters of the circle.
The ratio of the circle surface $\left(P_{C I R C L E}\right)$ and the frame $\left(P_{\text {FRAME }}\right)$ is

$\frac{P_{C I R C L E}}{P_{\text {FRAME }}}=\frac{r^{2} \pi}{4 r^{2}}=\frac{\pi}{4}=0,785$,

A similar mathematical calculation procedure can be used for area ratios for other road signs. For other shapes, the characteristic occupancy values are:

- triangle - the value is 0,5 ;

- $\quad$ octagon - the value is $\sim 0,83$;

- $\quad$ square and rectangle - the value is 1 .

In practice, the size of traffic signs slightly deviates from the regular geometric shapes. For example, the triangle signs have rounded corners and will take a little more area inside the frame (approximately 0,55 ).

Shape parameter values depend on the view angle and are to be taken into consideration when analysing the traffic signs to define the boundaries. Their graphical representations are given in Fig. 11 and Fig. 12. These diagrams show a reduction of the bounding box ratio, Fig. 11 , as well as the ratio of the sign area and the bounding box area as the viewing angle increase, Fig. 12.

At the time of collecting parameter values, the rectangular traffic sign is not accessible for the analysis. Therefore, it was not possible to perform experimental measurement and displayed graphical results for it.

The data from the graphs enable the creation of the border search of the object shape based on those properties. The graph (Fig. 11) shows that the signs are at approximately value "1" at angle $0^{\circ}$. Basically, the width and height of the bounding box have a similar amount.

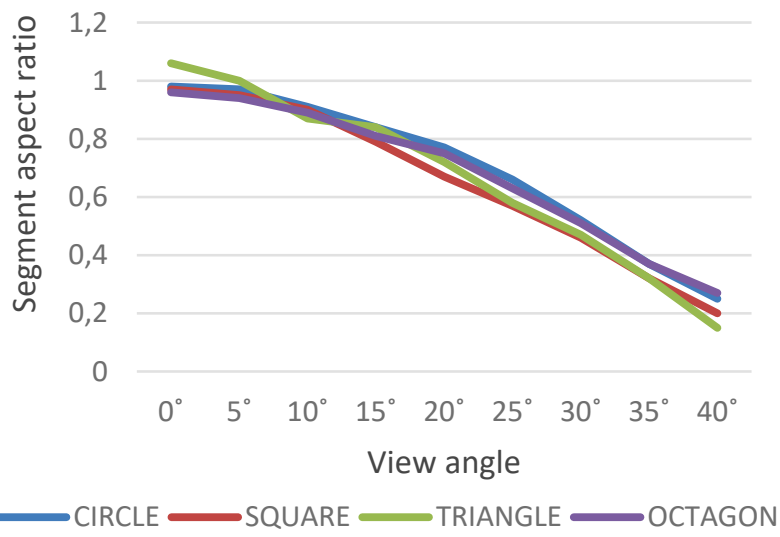

Figure 11 Frame ratio in different viewing angles

Knowing the dimensions of the rectangular signs for the second shape parameter, its value should be around 66 $\%$ at the same angle of $0^{\circ}$. It is, therefore, necessary to evaluate whether to include rectangular signs in the range of the values because simply by extending the range, we expand the space for false detection of candidates of the binary image.

One of the acceptable ranges for the first shape parameters ranges from 0,5 to 1,2 . It comprises a value up to $30^{\circ}$ angle of view of the traffic signs illustrated in Fig. 11. 
The graph (Fig. 12) shows the second shape parameters which are required for the classification of the traffic signs according to the form pursuant to which the objects pass the authentication of the frame aspect ratio. The chart shows that the values of the circle and octagon are approximate and they are, therefore, classified in one group of traffic signs. It is also evident that the value of the triangular traffic sign ranges from $55 \%$ to $45 \%$ as well as from $97 \%$ to $70 \%$ for the square. The circle or octagon range from $83 \%$ to $68 \%$. With the increase of the traffic sign viewing angle, it is realistic to expect that the recognition will be a lot more difficult to implement, if not impossible. Hence, to take a realistic range of values for the triangular sign, we use interval $[0,45 \div 0,60]$, for the circular sign interval $[0,6 \div 0,85]$ and for the square sign interval $[0,85 \div 1]$. The values are estimated for all intervals and some intervals increase due to the human factor when collecting data.

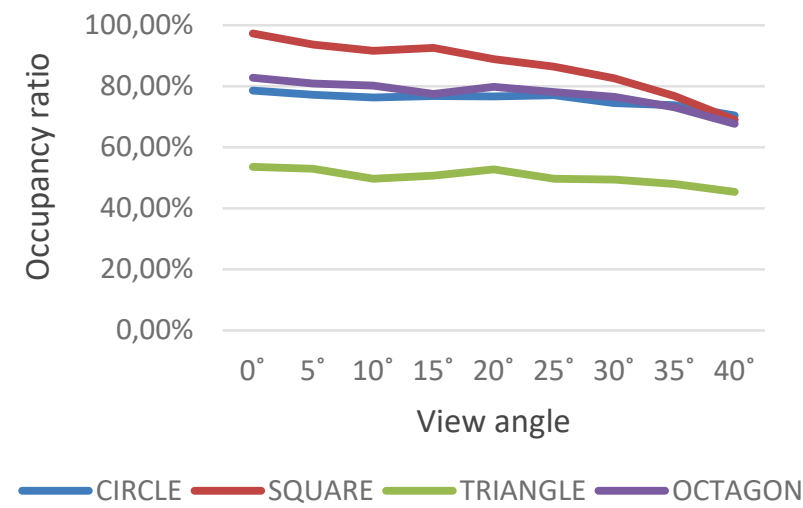

Figure 12 Sign shapes surface occupancy change vs. viewing angle

Fig. 13 gives an overview of the realistic traffic sign example under different viewing conditions, angles $0^{\circ}$ to $40^{\circ}$. This figure illustrates the difficultness in detection and content analysis of the analysed sign shape due to shape shifting and image distortion overall.

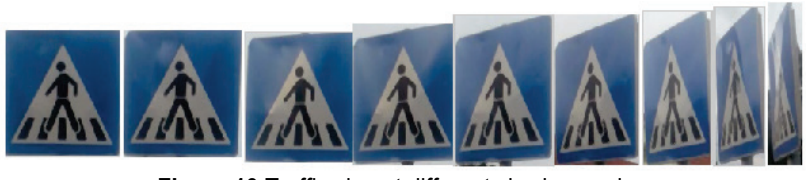

Figure 13 Traffic sign at different viewing angles

\subsection{Content Recognition}

The recognition phase covers the part of the programme preparation of an image for the comparison with given templates. In order for the comparison to take place, it is necessary to adjust the size of candidate images to those in the template. We use the nearestneighbour interpolation method for that. The sizes of templates are magnitude of $64 \times 64,128 \times 128$ and $256 \times 256$ pixels and to those sizes the input binary images are to be adjusted prior to further analysis steps.

In the implementation of recognition, the input binary image is adjusted to each size of the templates magnitude. Further, 2D correlation analysis of the same magnitude template and object images follows. After we get the correlation values for all three sizes, the largest one is taken into account. The last step of the recognition phase is drawing the bounding box on positively identified candidates of the traffic signs in the image with the percentage of the correlation coefficient. With the correlation, we measure numerical linear dependence between two variables. The number sign of the correlation depends strictly on the numerator whose multiplication gets positive or negative values.

The correlation result represents the correlation coefficient " $r$ " ranging from " -1 " to " $1 "$. As the result gets closer to " 1 " or "1", the variables are more associated. If the correlation coefficient is close to " -1 ", it is a negative correlation. Consequently, the relationship among variables is established, i.e. if one variable value rises, the other one falls. If the correlation coefficient is close to "1", the value of both variables goes up or down. In the case where the correlation coefficient value is close to " 0 ", there is no correlation between the variables.

The areas of interest for us are the values that are close to "1", where "1" corresponds to the perfect correlation between two variables, in this case, two images. The mathematical equation of the $2 \mathrm{D}$ correlation coefficient is given by the expression

$r=\frac{\sum_{m} \sum_{n}\left(X_{m n}-\bar{X}\right)\left(Y_{m n}-\bar{Y}\right)}{\sqrt{\sum_{m} \sum_{n}\left(X_{m n}-\bar{X}\right)^{2}\left(Y_{m n}-\bar{Y}\right)^{2}}}$,

$X$ and $Y$ images we are comparing and the subscript indices $\mathrm{m}$ and $\mathrm{n}$ refer to the pixel location in the image. $\bar{X}$ and $\bar{Y}$ are the mean intensity of the whole first and second image. Basically, the equation calculates every pixel location in both image double-sum difference between the intensity value at that pixel and the mean intensity of the whole image.

\section{TESTS AND RESULTS}

The programme implementation was carried out in the MATLAB programming environment with the installed software tools for image processing (Image Processing Toolbox).

The parameters that are used in the implementation of the testing program are as follows.

- Threshold parameters:

\section{Binary image of red colour $=$ $\left\{\begin{array}{c}\text { Red channel }>\text { treshold } \\ \text { Green channel }<k 1 * \text { Red channel }, \\ \text { Blue channel }<k 2 * \text { Red channel }\end{array}\right.$}

$$
\begin{aligned}
& \text { Binary image of blue colour }= \\
& \text { Blue channel }>\text { treshold } \\
& \text { Red channel }<k 3 * \text { Blue channel, } \\
& \text { Green channel }<k 4 * \text { Blue channel }
\end{aligned}
$$

- $\quad$ threshold $=40, k 1=0,62, k 2=0,67, k 3=0,80, k 4=0,57$

- The condition of a minimum of 0,25 correlation coefficient in order to traffic sign candidates can be classified and recognized.

- Parameter size of the structural elements: $S E=4$

- Incorrectly recognised traffic signs

- Falsely recognised traffic signs - recognised as road sign objects when in fact they are not. 
- Unrecognised traffic signs.

- The average correlation coefficient - share of the total number of recognised traffic signs with the number of traffic signs which are seen (not including those which were not recognised for some reason).

Coefficients $k 1, k 2, k 3$ and $k 4$ and threshold are determined empirically in order to give the best segmentation outcomes as possible. They are purely numerically expressed and suit to images with good as well as to images with limited light conditions (they stay fixed for all tested pictures). Table 1 shows a range where $k$ parameters have their best application results. For our tested pictures, the optimum values are listed above.

Table 1 Optimum coefficients of $k$ parameters

\begin{tabular}{|c|c|c|}
\hline Coefficients & Min & Max \\
\hline $\mathrm{k} 1$ & 0,59 & 0,65 \\
\hline $\mathrm{k} 2$ & 0,64 & 0,70 \\
\hline $\mathrm{k} 3$ & 0,76 & 0,84 \\
\hline $\mathrm{k} 4$ & 0,54 & 0,60 \\
\hline
\end{tabular}

After applying the parameters, the test is carried out The following results are given and classified in four main categories: correctly (not shown in tables below), incorrectly recognised, falsely recognised and unrecognised signs.

Table 2 Recognition of blue traffic signs

\begin{tabular}{|l|c|c|c|}
\hline \multicolumn{1}{|c|}{ Blue TS } & Circular TS & Square TS & Total \\
\hline Number of TS & 13 & 9 & 22 \\
\hline Incorrectly recognised TS & 0 & 0 & 0 \\
\hline Falsely recognised TS & 0 & 0 & 0 \\
\hline Unrecognised TS & 1 & 0 & 1 \\
\hline Average correl. coefficient & 0,7608 & 0,4578 & 0,6309 \\
\hline
\end{tabular}

Table 3 Recognition of red traffic signs

\begin{tabular}{|l|c|c|c|}
\hline \multicolumn{1}{|c|}{ Red TS } & Circular TS & Triangle TS & Total \\
\hline Number of TS & 33 & 14 & 47 \\
\hline Incorrectly recognised TS & 1 & 2 & 3 \\
\hline Falsely recognised TS & 0 & 1 & 1 \\
\hline Unrecognised TS & 2 & 0 & 2 \\
\hline Average correl. coefficient & 0,7730 & 0,7062 & 0,7528 \\
\hline
\end{tabular}

Table 4 Recognition of traffic signs in reduced visibility

\begin{tabular}{|l|c|}
\hline \multicolumn{1}{|c|}{ TS in reduced visibility } & Total \\
\hline Number of TS & 18 \\
\hline Incorrectly recognised TS & 1 \\
\hline Falsely recognised TS & 1 \\
\hline Unrecognised TS & 4 \\
\hline Average correlation coefficient & 0,7042 \\
\hline
\end{tabular}

As can be seen from the tables (Tab. 2, Tab. 3, Tab. 4) and graphs (Fig. 14, 15, 16), in the conditions of good visibility when the separation of colours can be very easily detached, the segmentation process achieves a considerable success in the detection, classification and recognition of traffic signs (over $90 \%$ correct recognition on a set of testing pictures).

In the case of blue sign candidates, in only one case the traffic sign failed to pass the stage classification because the segmentation fit into the environment of the similar colour.

In the case of red sign candidates, we have several situations where the traffic sign experienced a difficulty to be positively identified. Some situations of incorrectly recognised traffic signs refer to cases when the sign surface is covered with dirt. Also, we have a case of merging with colour similar to the area of the traffic sign and cases of not recognising the traffic sign due to the interference with external environment. One case corresponds to the false recognition of the traffic sign object with a database which is not a traffic sign.

\section{EFFICIENCY OF BLUE TS RECOGNITION}

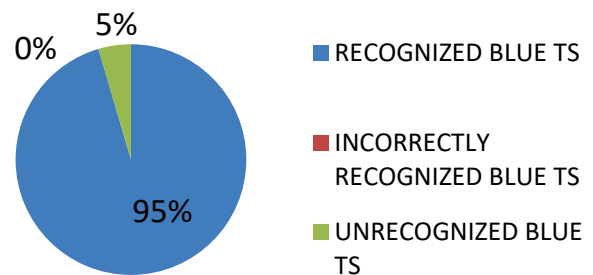

TS

Figure 14 Percentage of blue recognised traffic signs

\section{EFFICIENCY OF RED TS RECOGNITION}

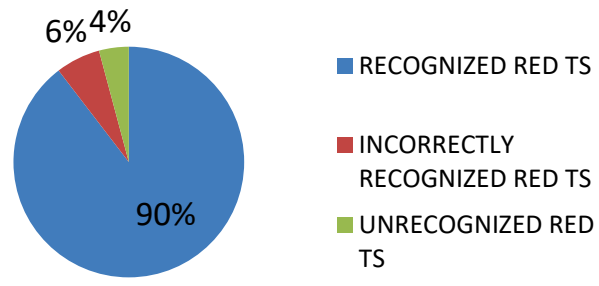

Figure 15 Percentage of red recognised traffic signs

\section{EFFICIENCY OF TS IN REDUCED VISIBILITY}

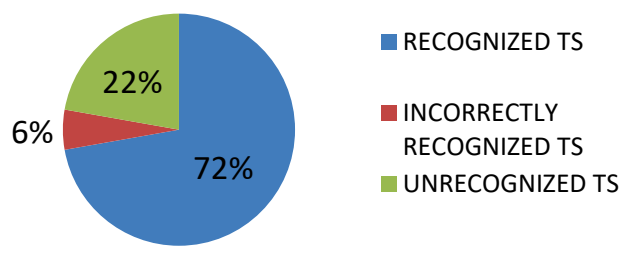

Figure 16 Percentage of recognised traffic signs in reduced visibility

In the case of application in the reduced visibility environment, this method shows its weakness and reduced performance overall. In numerous situations, we come to partial detection of the sign candidate colours, thus making them not enough spatially visible for the successful recognition. It reveals a major flaw of the RGB system which is very sensitive to intensity values and in these cases, it is not as robust as a system because the slightest change in the value of lighting can crash the whole concept of proposed traffic sign recognition. In all three mentioned cases, the correlation coefficient on average is in quite a high interval [0,63 to 0,75$]$.

Furthermore, we review the application results of the traffic sign recognition method under a different viewing angle, distances and inclination of the traffic sign. Fig. 17 illustrates an example of traffic sign B28 correlation movement depending on the viewing angle. Despite the fact that the viewing angle is much wider, traffic sign B28 is positively identified from the database even with a high drop in the correlation coefficient from 0,93 to 0,56 . It can be concluded that the decrease in the correlation 
coefficient increases as the viewing angle increases. The result is expected because the road sign, when viewed at an angle, takes a substantially different form of shapes than a template from the database.
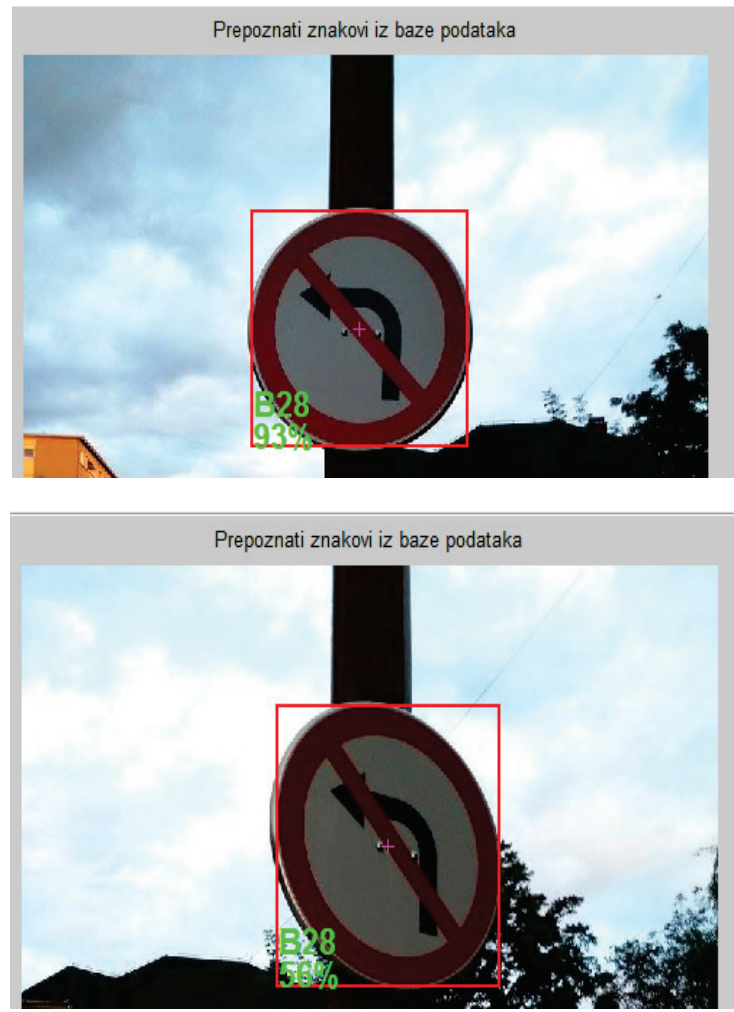

Figure 17 Traffic sign recognition at a different view angle

Fig. 18 demonstrates an example of a correlation movement depending on the distance of traffic sign B57. The drop of correlation is not so substantial compared to an example of a wider viewing angle. The correlation coefficient on the current example traffic sign changes from 0,91 to 0,82 , which is a slight decrease, and the sign was detected properly in the database.

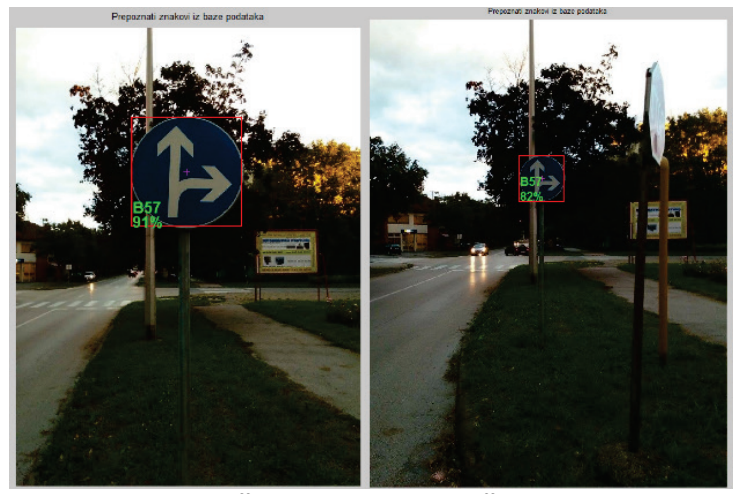

Figure 18 Traffic sign recognition at different distances

As can be seen in Fig. 19, the example of A20 traffic sign shows the inclination influence on a much greater level of effect on the correlation coefficient than a distance of traffic sign dropping from 0,8 to 0,64 due to a slight inclination. This happens due to the increase of the distance shape of the sign which will still hold approximately similar shape with the traffic sign from the database with some data loss due to the increasing distance.
An increase in inclination leads to discrepancies of a shape with the shape of the template; therefore, a drop in correlation is quite significant. The sign was properly identified in the database.

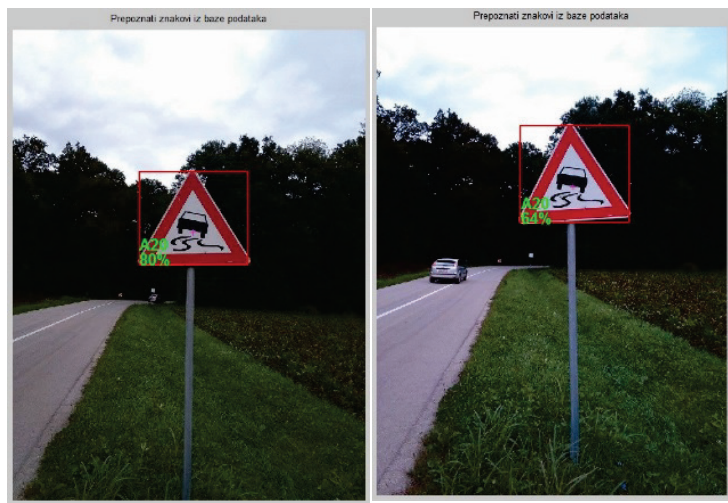

Figure 19 Traffic sign recognition at different inclinations

\section{CONCLUSION}

Based on the experimental results, we can assert that the process of recognition of traffic signs is satisfactory regarding the number of detected traffic signs. When the brightness of traffic signs is at a satisfactory level, the programme works extremely satisfactory even in rotated situations and at greater distances of traffic signs. During the experimenting, we noticed that the angle of inclination to the left or right side and the angle of view to a sign have a lot of influence in terms of the correlation coefficient drop. Although the decrease in the coefficient of similarity was significant, it was still possible to recognise the traffic sign by comparing it with the template database of traffic signs. Hence, we can conclude that the correlation and template comparison method are not robust methods in terms of variation of the viewing angle and inclination; yet, they are still satisfactory methods that achieve the goal of successfully identifying the traffic sign. In conditions when the light source is limited, for example, early evening, night, or when the sign is located in shadows, there is a significant decrease in the performance of traffic signs recognition. The problem lies in the fact that the RGB colour model is not robust in terms of variations of light. Consequently, the improvement of the programme should be sought in other models of colour that separate the colour information from the information intensity.

This method and the shape recognition approach have neither been optimised for fast computation nor is the performance analysis conducted. This happens because, at this moment, the main objective is set to prove the method applicability as a successful traffic sign detector and recogniser. The detection results are satisfactory and are in the middle compared to the methods overviewed in [7]. So, as future work, this method can be implemented in a more efficient way by using more performance convenient programming environment. Also, a detailed performance analysis and suitability for a real time application can be carried out and compared to other methods regarding accuracy and execution time.

Finally, the test and analysis results prove, to some extent, the applicability and usability of the proposed method in applications for traffic signs detection and 
recognition as well as confirm the validity of the method potential. We achieve the set goal with the segment surface occupancy analysis approach as it did an excellent job with very good results in the traffic sign recognition process.

\section{REFERENCES}

[1] Escalera, S., Baro, X., Pujol, O., Vitrià, J., \& Radeva, P. (2011). Traffic - Sign Recognition Systems. Springer. https://doi.org/10.1007/978-1-4471-2245-6

[2] Fleyeh, H. (2008). Traffic and Road Sign Recognition. PhD Thesis, Napier University, Edinburgh, Great Britain.

[3] Greenhalgh, J. \& Mirmehdi, M. (2012). Real-Time Detection and Recognition of Road Traffic Signs. IEEE Transaction on Intelligent Transportation Systems, 13(4), 1498-1506. https://doi.org/10.1109/TITS.2012.2208909

[4] Broggi, A., Cerri, P., Medici, P., Porta, P., \& Ghisio, G. (2007). Real time road signs recognition. IEEE Intelligent Vehicles Symposium / Turkey, June 2007, 981-986. https://doi.org/10.1109/IVS.2007.4290244

[5] Nguwi, Y. \& Kouzani, A. Z. (2008). Detection and classification of road signs in natural environments. Neural Computing and Applications, 17(3), 265-289. https://doi.org/10.1007/s00521-007-0120-z

[6] Mogelmose, A., Trivedi, M. M., \& Moeslund, T. B. (2012). Vision-based traffic sign detection and analysis for intelligent driver assistance systems: Perspectives and survey. IEEE Transactions on Intelligent Transportation Systems, 13(4), 1484-1497. https://doi.org/10.1109/TITS.2012.2209421

[7] Mathias, M., Timofte, R., Benenson, R., \& Gool, L. V. (2013). Traffic Sign Recognition - How far are we from the solution? International Joint Conference on Neural Networks / Dallas, USA, August 2013, 1-8. https://doi.org/10.1109/IJCNN.2013.6707049

[8] Benallal, M. \& Meunier, J. (2003). Real-time color segmentation of road signs. IEEE CCECE 2003, Canadian Conference on Electrical and Computer Engineering, 3, 1823-1826. https://doi.org/10.1109/CCECE.2003.1226265

[9] Baro, X. \& Vitria, J. (2004). Fast traffic sign detection on greyscale images. Recent Advances in Artificial Intelligence Research and Development. Frontiers in Artificial Intelligence and Applications, 113, pp. 69-76.

[10] Rules on traffic signs, signalling and equipment on the roads. http://www.instruktor-voznje.com.hr/pdf/pravilnik_o prometnim_znakovima.pdf (25 July 2017)

[11] Abedin, Z., Dhar, P., Hossenand, M. K., \& Deb, K. (2017). Traffic Sign Detection and Recognition Using Fuzzy Segmentation Approach and Artificial Neural Network Classifier Respectively. International Conference on Electrical, Computer and Communication Engineering, ECCE / Cox's Bazar, Bangladesh, Feb. 2017, 518-523. https://doi.org/10.1109/ECACE.2017.7912960

[12] Ardianto, S., Chen, C. J., \& Hang, H. M. (2017). Real-Time Traffic Sign Recognition using Color Segmentation and SVM. International Conference on Systems, Signals and Image Processing, IWSSIP / Poznan, Poland, July 2017, 15. https://doi.org/10.1109/IWSSIP.2017.7965570

[13] Paulo, F. C. \& Correia, P. L. (2008). Traffic Sign Recognition Based on Pictogram Contours. Ninth International Workshop on Image Analysis for Multimedia Interactive Services, WIAMIS / Klagenfurt, Austria, 67-70. https://doi.org/10.1109/WIAMIS.2008.31

[14] Qian, R., Yue, Y., Coenen, F., \& Zhang, B. (2016). Traffic Sign Recognition with Convolutional Neural Network Based on Max Pooling Positions. 12 th International Conference on Natural Computation, Fuzzy Systems and Knowledge Discovery / Changsha, China, Aug. 2016, 1-5.
https://doi.org/10.1109/FSKD.2016.7603237

[15] Forczmański，P. (2013). Recognition of occluded traffic signs based on two-dimensional linear discriminant analysis. Archives of Transport System Telematics, 6(3), $10-13$.

\section{Contact information:}

Tomislav KESER, Assistant Professor

J. J. Strossmayer University of Osijek,

Faculty of Electrical Engineering, Computer Science and Information Technology Osijek

Cara Hadrijana 10b, Osijek, Croatia

tomislav.keser@ferit.hr

\section{Ivan DEJANOVIĆ, BEng}

J. J. Strossmayer University of Osijek

Faculty of Electrical Engineering, Computer Science and Information Technology Osijek

Cara Hadrijana 10b, Osijek, Croatia

idejanov@gmail.com 